\title{
Multiscale Models in Heterogeneous Catalysis: Incorporating Dynamics into
}

Reaction Kinetics

Toyin Omojola*

*toyin.omojola@cantab.net

\section{Abstract}

Modern operando spectroscopy and microscopy, and kinetic investigations have provided qualitative evidence for active site dynamics, catalyst surface dynamics and charge transport. On the macroscale, intraparticle and interparticle mass and heat transfer can be tuned to optimise selectivity over heterogeneous catalysts. On the microscale, adsorbate-induced restructuring, adsorbate mobility, surface composition, oxidation states, charge transport, band gap, and the degree of coordination of the active site have been identified for controlling product selectivity. There exists, however, limited physics-based and data-driven multiscale models that can assimilate these qualitative descriptors in an integrated manner to extract quantitative catalyst activity, stability and product selectivity descriptors. A multiscale model, which describes the evolution of gas species, adspecie accumulation due to reactivity, stability, lifetime, and mobility, charge transport involving electrons and holes, heat transfer for non-isothermal conditions due to reaction exothermicity, and the changing catalyst states is provided. Dynamical effects are included into these models to bridge the gap between laboratory scale studies and industrial technical reactors.

Keywords: dynamics, reaction engineering, heterogeneous catalysis 


\section{Introduction}

Rapid developments in the use of characterisation techniques to observe individual catalytic turnovers at the atomic scale have led to huge progress in the use of microscopy ${ }^{1-3}$, structural characterization, and data handling ${ }^{4}$ to provide clarity on the dynamics of particle shapes and sizes, catalyst surfaces on each facet of the particle shape, and the local environment of the active site. Correspondingly, there has been a parallel evolution in molecular computational catalysis to quantify the aforementioned operando studies. ${ }^{5}$

On the molecular level, selectivity over mixed metal oxides depends on the catalyst surface structure ${ }^{6-8}$, catalyst shape and size $^{9}$, and active site dynamics ${ }^{10}$. Selectivity depends on adsorbate-induced restructuring, adsorbate mobility, the reactivity of reaction intermediates, surface composition, oxidation states, and charge transport. ${ }^{11}$ Product selectivity can only be established if the reaction network is determined. Selectivity can be tuned, on the macroscale level, by careful adjustment of intraparticle and interparticle heat and mass transfer. ${ }^{12}$ Other factors include the degree of coordination of the active site, band gap of the oxide, acid-base properties, chemical bonding, defect sites, site-isolation and domain size, water influence, metal oxide support and ligand effects. ${ }^{13}$ Grasselli further postulated seven descriptors such as lattice oxygen, metal-oxygen bond, host structure, redox capability, multifunctionality of active sites, site isolation, and phase cooperation that could control catalyst design and synthesis. ${ }^{14}$

Various forms of dynamics could be experienced during heterogeneous catalysis. First, nanoparticles could exhibit overlayer formation or periodic reconstructions during catalytic reactions which prevents the use of the standard model of catalysis. ${ }^{15}$ Second, the local environment along the axial length of a fixed bed 
reactor has an intricate connection to the catalyst state and performance. ${ }^{16-18}$ Third, the nature of the active site could change upon chemisorption due to geometric (ensemble) and electronic (ligand) effects. ${ }^{19,20}$ Fourth, mobility of active sites could occur at elevated temperatures ${ }^{10}$. The changing nature and mobility of active sites during catalysis leads to substantial changes in quantity of active sites.

The changing catalyst surface (due to overlayer formation and periodic reconstructions), local environment (differential or integral reactor conditions), and quantity of active sites (nature, mobility) all influence of the adsorption constant of educts, intermediates, and products during oxidation catalysis. Consequently, the adsorption constant of each specie is related in a complex fashion to its coverage, the coverage of the most abundant surface specie during overlayer formation, time on stream due to aperiodic or periodic reconstructions ${ }^{21}$ and the local chemical potential.

Although, molecular and macroscopic factors that control selectivity have been distinguished qualitatively, there exists no quantitative framework in terms of datadriven physical models that can capture the entire range of complexity experienced from atomic level to reactor level. Focusing on oxidation catalysis, many studies have investigated surface and shape dynamics ${ }^{22-25}$. Using state-of-the-art surface science techniques, the catalyst composition during operando conditions have been elucidated. For instance, during the selective oxidation of propane over MoVTeNbOx catalysts, enrichment of the catalyst surface by tellurium has been observed in the presence of steam. ${ }^{26,27}$ Surface enrichment has also been observed during oxidative dehydrogenation of propane over $\mathrm{MnWO}_{4}$ nanorods ${ }^{28}$ and selective oxidation of propylene to acrolein over multi-component BiMo catalysts. ${ }^{29}$ Although an increasing effort is placed on the use of ab-initio density functional theory calculations to represent 
the changing nature and mobility of the active site during catalytic reactions ${ }^{10,19}$, there are limited integrated approaches which incorporate dynamical effects.

At this juncture, it is important to clarify what we mean by dynamics. Active site dynamics is not a new phenomenon in catalysis. Indeed, Thomas and Thomas ${ }^{12}$ provided extensive details on dynamic modelling during catalyst deactivation. Ert| ${ }^{30}$,

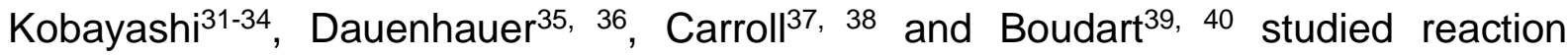
dynamics. Warshel ${ }^{41-45}$ investigated the dynamic phenomena during enzyme catalysis and observed that the duration of the kinetics of catalytic transformation is much smaller than conformational dynamics. In this paper, dynamics during heterogeneous oxidation catalysis is emphasised with respect to catalyst surface changes, local chemical potentials, and changing nature and mobility of active sites.

During oxidation catalysis over mixed metal oxide catalysts, active site representation is particularly complex due to multiple atoms and intricate functionalities available on the terminated catalyst surface. Surface metal bonds are relatively unsaturated compared to cations in the catalyst bulk. Several complexities are experienced during oxidation catalysis over mixed metal oxide catalysts. These include: (1) intricate reaction network, (2) multifunctionality of the different metal cations on the catalyst surface, (3) the various oxygen species which further expands the possible pathways in the reaction network, (4) changing active sites, (5) charge transport, and the (6) evolving catalyst surface. Due to the changing nature of the active sites and catalyst surfaces, a set of physics-based and data-driven multiscale computational models for heterogeneous catalysis could be assembled to obtain quantitative descriptors.

A systematic approach considering active site dynamics, surface dynamics and charge transport phenomena is followed in this work. First, active site dynamics due to 
the changing nature, mobility, and quantity of the active sites are investigated. This approach is particularly useful for selective oxidation reactions over multi-functional and multi-component mixed metal oxide catalysts where representations of the active site is challenging, albeit to which active site dynamics have been observed from modern operando characterisation methods such as transmission electron microscopy and other spectroscopic techniques. ${ }^{26,27,46,47}$ In order to understand active site dynamics, open catalytic cycles (single, and multiple) are proposed. Second, catalyst surface dynamics due to changing surface composition and termination, metal ion coordination, and the evolving different site distribution is provided. Wulff construction method $^{48,49}$ is typically used to provide equilibrium catalyst structures in density functional theory calculations. However, a revision of an old concept called "homotattic patch models" was sufficient enough to incorporate catalyst surface dynamics. Third, due to the oxygen reduction mechanism and charge transfer to the catalyst surface, charge transport is considered.

The set of models presented here should, at a minimum, describe the molecular and macroscopic factors that govern activity, stability, and selectivity during catalysis. ${ }^{11-13,50}$ These models consider not only elementary reaction steps, but accumulation of surface species, changing catalyst state and charge transport. 


\section{Active Site Dynamics}

A useful starting point for the incorporation of active site dynamics into reaction kinetics is to revisit the concept of closed catalytic cycles. The influence of facile adsorption is decoupled from changes due to electronic or geometric factors as given in scheme 1:

1. $A+* \rightleftharpoons A *-$ - - Facile adsorption on a static surface

2. $A * \rightleftharpoons A \llbracket$ - - Formation of an adsorption complex due to dynamicity The traditional concept of a catalytic cycle, based on static sites, is given as: ${ }^{13}$

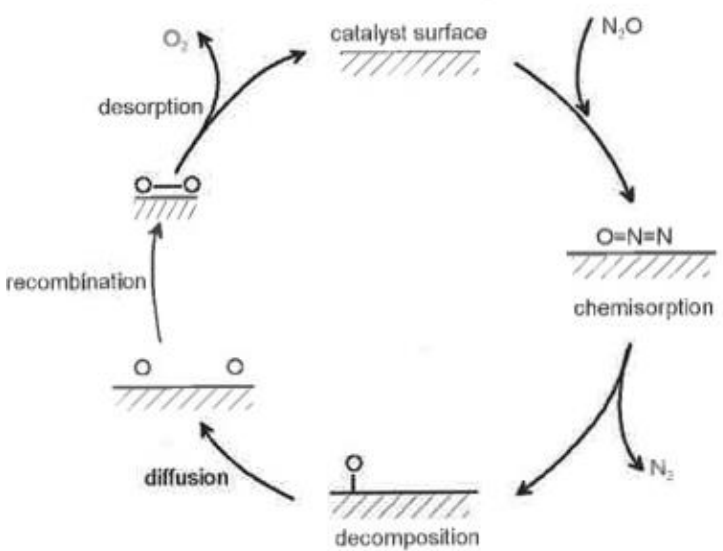

Figure 1: Schematic illustration of the catalytic cycle of reaction events for the decomposition reaction of $\mathrm{N}_{2} \mathrm{O}$ (closed catalytic cycle). Image reproduced from Ref. ${ }^{13}$

The active site dynamics concept leads to:

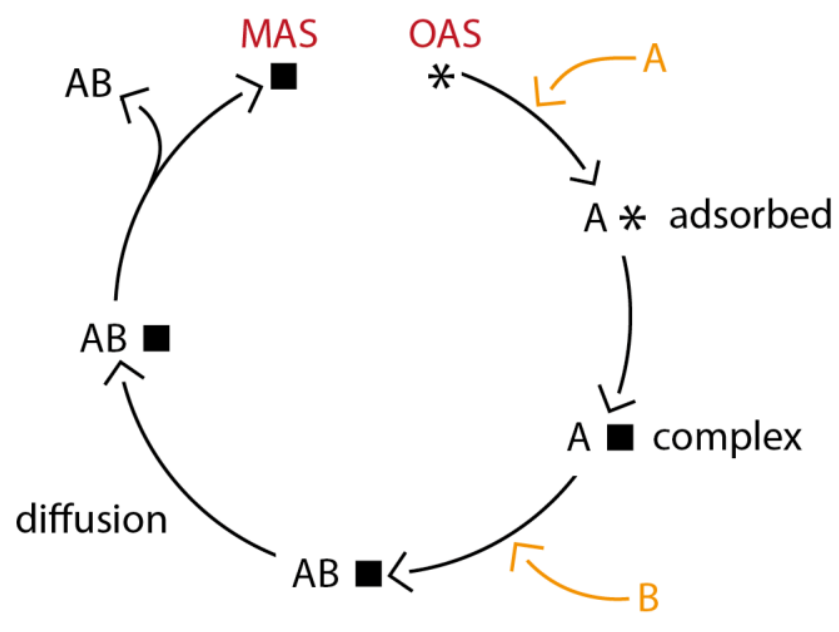

Figure 2: Schematic illustration on an open catalytic cycle of reaction events that considers active site dynamics 
In the revised concept of an open cycle, the adsorption process $\left(A^{*}\right)$ on the original active site (OAS) is decoupled from electronic or geometric modifications ( $\left.A^{-}\right)$. Subsequent reactions occurs where $B$ specie reacts with $\left(A^{-}\right)$to form $(A B-)$. Following diffusion, desorption occurs such that a modified active site (MAS) is obtained, which could be involved in the next cycle (Figure 3).

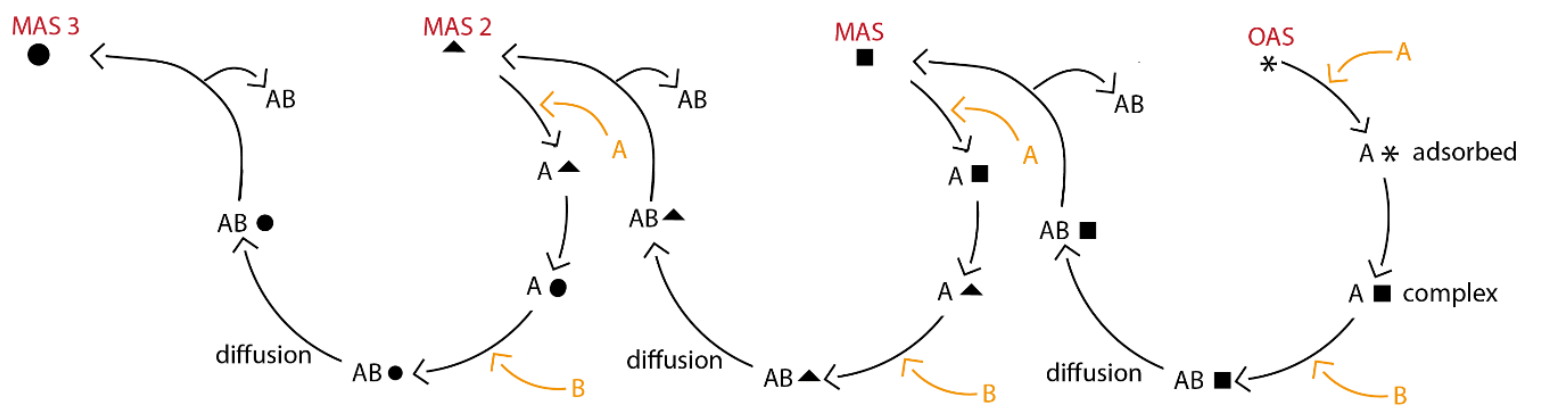

Figure 3: A series of consecutive open catalytic cycles onto which the same reactants and products are formed

Eventually in the absence of deactivation, multiple open catalytic cycles may lead to a closed catalytic cycle (Figure 4).

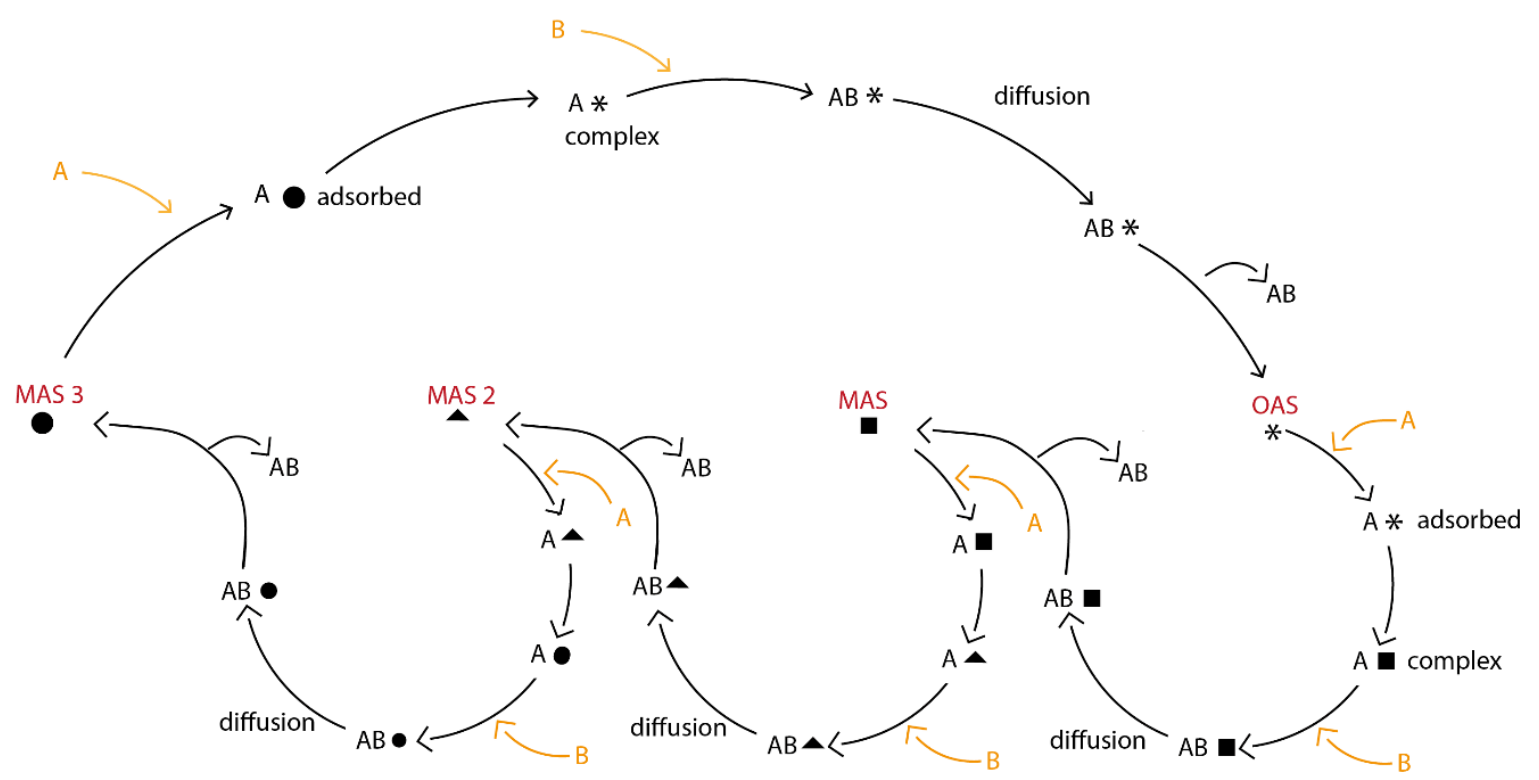

Figure 4: A series of consecutive open catalytic cycles leading finally to an open catalytic cycle during which reactants and products are formed 
In Figures 2 - 4, the original active site (OAS) could be quantified using, e.g., pyridine Fourier Transform Infrared Spectroscopy. For open catalytic cycles, the OAS changes on each catalytic turnover to MAS and so on. Consequently, a titration of MAS is required. Boudart ${ }^{51}$ defined the turnover frequency as the molecules converted by catalytic active site per second. Consequently, in one second, it is the number of molecules converted by a catalytic active site, assuming of course that this site retains its nature. However, if the catalytic active site changes its nature due to the adsorption of molecules, then a re-definition is required, and quantification is provided.

\subsection{Quantification: Active site dynamics}

The revised concept of an open catalytic cycle requires that the modified active sites (MAS) after adsorption of a specie is titrated. During an open catalytic cycle, the number of original active sites change to modified active sites with each turnover.

In the closed catalytic cycle concept, we start with a basic assumption: that the quantity of the OAS is a root (i.e., a solution) of the function of the number of active sites with each catalytic turnover time. Subsequent cycles in the closed catalytic cycle concept ensures that one root in the relationship between number of active sites and turnover is obtained and maintained. In the open catalytic cycle concept, however, we could envisage that multiple roots in that functional relationship between the number of active sites and turnover are obtained. Furthermore, each catalytic cycle could be an attempt to return to the original root of this relationship or diverge from this original root. Consequently, the Newton and Raphson ${ }^{52}$ iterative equation suffices to obtain that convergence towards the original root. Another relationship may be used to obtain the divergence from the original root of this functional dependence. In our 
consideration below, we use the Newton-Raphson iterative equation to restrict our analysis towards the convergence towards the original root.

For one open catalytic cycle, we obtain:

$$
M A S=O A S-\frac{f(O A S)}{f^{\prime}(O A S)_{O}}
$$

For multiple open catalytic cycles, we obtain:

$$
\begin{aligned}
& M A S_{2}=M A S-\frac{f(M A S)}{f^{\prime}(M A S)_{o}} \\
& M A S_{3}=M A S_{2}-\frac{f\left(M A S_{2}\right)}{f^{\prime}\left(M A S_{2}\right)_{o}} \\
& M A S_{4}=M A S_{3}-\frac{f\left(M A S_{3}\right)}{f^{\prime}\left(M A S_{3}\right)_{o}} \\
& M A S_{n}=M A S_{(n-1)}-\frac{f\left(M A S_{(n-1)}\right)}{f^{\prime}\left(M A S_{(n-1)}\right)_{o}}
\end{aligned}
$$

For a finite series, we obtain:

$$
\begin{aligned}
& M A S_{4}=O A S-\frac{f(O A S)}{f^{\prime}(O A S)_{o}}-\frac{f(M A S)}{f^{\prime}(M A S)_{o}}-\frac{f\left(M A S_{2}\right)}{f^{\prime}\left(M A S_{2}\right)_{o}}-\frac{f\left(M A S_{3}\right)}{f^{\prime}\left(M A S_{3}\right)_{o}} \\
& M A S_{i+1}=O A S-\sum_{i=0}^{3} \frac{f\left(M A S_{i}\right)}{f^{\prime}\left(M A S_{i}\right)_{o}}
\end{aligned}
$$

For an infinite series, this is equal to:

$$
M A S_{n+1}=O A S-\int_{0}^{n} \frac{f\left(M A S_{n}\right)}{f^{\prime}\left(M A S_{n}\right)_{o}} d\left(M A S_{n}\right)
$$

By integration using u-substitution for $f\left(M A S_{n}\right)$, we obtain:

$$
\begin{aligned}
& M A S_{n+1}=O A S-\int_{0}^{n} \frac{u}{d u} d u=O A S-\left[\frac{u^{2}}{2}\right]_{0}^{n}=O A S-\frac{n^{2}}{2} \\
& M A S_{n+1}=O A S-\frac{f\left(M A S_{n}\right)^{2}}{2}
\end{aligned}
$$


The solution to the open catalytic cycle proposed in Equation 2.10 shows that the number of modified active sites of an $(n+1)$ th cycle can be titrated using the original active sites and the function of modified active sites of an nth cycle during the catalytic turnover involving adsorption, desorption, diffusion, and reaction of species. The main challenge is the titration of the modified active sites which is dependent on the turnover. Finally, for multiple open catalytic cycles leading to a closed catalytic cycle, we obtain:

$$
\begin{aligned}
& M A S=O A S-\frac{f(O A S)}{f^{\prime}(O A S)_{o}} \\
& M A S_{2}=M A S-\frac{f(M A S)}{f^{\prime}(M A S)_{o}} \\
& M A S_{3}=M A S_{2}-\frac{f\left(M A S_{2}\right)}{f^{\prime}\left(M A S_{2}\right)_{o}} \\
& M A S_{4}=M A S_{3}-\frac{f\left(M A S_{3}\right)}{f^{\prime}\left(M A S_{3}\right)_{o}} \\
& O A S=M A S_{4}-\frac{f\left(M A S_{4}\right)}{f^{\prime}\left(M A S_{4}\right)_{o}}
\end{aligned}
$$

For a closed finite cycle, we obtain:

$$
\begin{aligned}
& O A S=O A S-\frac{f(O A S)}{f^{\prime}(O A S)_{o}}-\frac{f(M A S)}{f^{\prime}(M A S)_{o}}-\frac{f\left(M A S_{2}\right)}{f^{\prime}\left(M A S_{2}\right)_{o}}-\frac{f\left(M A S_{3}\right)}{f^{\prime}\left(M A S_{3}\right)_{o}}-\frac{f\left(M A S_{4}\right)}{f^{\prime}\left(M A S_{4}\right)_{o}} \\
& \frac{f(O A S)}{f^{\prime}(O A S)_{o}}=-\sum_{i=0}^{4} \frac{f\left(M A S_{i}\right)}{f^{\prime}\left(M A S_{i}\right)_{o}}
\end{aligned}
$$

For a closed infinite series, this is equal to:

$$
\frac{f(O A S)}{f^{\prime}(O A S)_{o}}=-\int_{0}^{n} \frac{f\left(M A S_{n}\right)}{f^{\prime}\left(M A S_{n}\right)_{o}} d\left(M A S_{n}\right)
$$

By integration using u-substitution for $f\left(M A E_{n}\right)$, we obtain:

$$
\frac{f(O A S)}{f^{\prime}(O A S)_{o}}=-\int_{0}^{n} \frac{u}{d u} d u=-\left[\frac{u^{2}}{2}\right]_{0}^{n}=-\frac{n^{2}}{2}
$$


$\frac{f(O A S)}{f^{\prime}(O A S)_{o}}=-\frac{f\left(M A S_{n}\right)^{2}}{2}$

Substituting this in 2.10 , we obtain:

$M A S_{n+1}=O A S-\frac{f(O A S)}{f^{\prime}(O A S)_{o}}$

Equation 2.18 implies that the modified active sites can be titrated based on the number density of the original active sites. A functional that describes the change of the number of original active site with one catalytic turn-over and a differential of that functional is required.

\section{Beyond the Standard Model of Catalysis}

The Langmuir model assumes a uniform surface characterized by a constant energy of adsorption (Figure 5a). The concept of biographical heterogeneity appeared over 80 years ago. Taylor ${ }^{53}$ examined metallic hydrogenation catalysts using X-rays. A terrace-ledge-kink (TLK) model of heterogeneous catalytic surfaces was proposed. ${ }^{51,54-56}$ The Langmuir model can be derived from kinetic or statistical mechanical considerations. From kinetic considerations, we obtain: 57

$$
f(\theta)=1-f^{\prime}(\theta)
$$

Where $f(\theta)$ and $f^{\prime}(\theta)$ represent, respectively, the fraction of surface that is vacant and the fraction of the vacant surface that is covered, say for instance by specie A. The fraction of molecules adsorbed of the total number of molecules striking a surface is:

$$
X(T, E)=\exp \left(-E / k_{B} T\right)
$$

Where $k_{B}$ is the Boltzmann constant and $E$ is the activation energy. The rate of adsorption then is:

$r_{a}=k_{a} \exp \left(-E / k_{B} T\right) f(\theta) p$ 
$k_{a}=\frac{s}{\left(2 \pi m k_{B} T\right)^{1 / 2}}$

Where " $\mathrm{s}$ " is the orientation factor to account for the fact that not all potentially successful encounters are actually successful. The rate of desorption is given by:

$r_{d}=k_{d} f^{\prime}(\theta) \exp \left(-E^{\prime} / k_{B} T\right)$

Where $k_{d}$ is the rate constant and $E$ is the activation energy of desorption. Equating the rate of adsorption to the rate of desorption, we obtain:

$\frac{f^{\prime}(\theta)}{f(\theta)}=K_{O}\left[\exp \left(Q / k_{B} T\right)\right] p$

$K_{O}=\frac{s}{k_{d}\left(2 \pi m k_{B} T\right)^{1 / 2}}$

$Q$ is the negative of the heat of adsorption and is given by $\left(E^{\prime}-E\right)$. When one adsorption site is required for each adsorbate molecule: $f(\theta)=1-\theta$ and $f^{\prime}(\theta)=\theta$ and thus,

$\theta=\frac{K p}{1+K p} ; K=K_{O}\left[\exp \left(Q / k_{B} T\right)\right]$

Following statistical mechanical considerations, Fowler and Guggenheim ${ }^{58}$ derived the isotherm assuming the molecules to be locally adsorbed on a square array of sites on a uniform surface without mutual interactions. The equation derived from statistical mechanical considerations is of the same form as from chemical kinetics, but " $K$ " has a more fundamental definition as the ratio of partition function of the adsorbed molecule to that of the gaseous molecules at infinite separation, with Ko given by:

$K_{O}=\left[\frac{h}{\left(2 \pi m k_{B} T\right)^{1 / 2}}\right]^{3}\left(\frac{q_{V}}{k_{B} T}\right)$

and $q_{v}$ being the vibrational partition function.

The Langmuir isotherm can also be expressed in terms of enthalpy and entropy functions: 59 
$\theta=\frac{1}{1+\left(b_{o} / p\right) \exp \left(-Q / k_{B} T\right)} ;$ or $p=b_{O}\left(\frac{\theta}{1-\theta}\right) \exp \left(-Q / k_{B} T\right)$

$b_{O}=e^{\Delta s^{*} / k_{B}} ; \Delta \mathrm{s}^{*}$ represents all entropy changes excluding configurational entropy.

We use the homotattic patch model ${ }^{60}$ to develop the fundamental equations for the overall isotherm for a surface with biographical heterogeneity leading to the adsorption integral equation. The homotattic patch model postulates different patches of different heats of adsorption to account for surface heterogeneity. The Langmuir isotherm for a single patch is integrated between selected upper and lower limits of the heat of adsorption for a given distribution of the adsorption heat. The homotattic patch model consists of sites grouped in patches where each patch contains a sufficiently large number of sites that can be regarded as a separate thermodynamic entity (Figure $5 b$ ). We consider the adsorption integral equation to represent such heterogeneity.

The Langmuir equation for a homogeneous surface when summed over $n$ patches is: ${ }^{57}$

$\theta_{m}(p)=\sum_{i} \frac{K p}{1+K p}$

Following statistical mechanical considerations, ${ }^{58}$

$K=\left[\frac{h}{\left(2 \pi m k_{B} T\right)^{1 / 2}}\right]^{3}\left(\frac{q_{V}}{k_{B} T}\right) \exp \left(\frac{Q}{k_{B} T}\right)$

$\theta_{m}$ in this equation is the overall isotherm for the entire surface, and $\mathrm{K}$ may be regarded as the ratio of the adsorbate to gas phase partition functions for the ith patch. The summation sign is replaced by an integration sign when the number of patches is large.

Equations 3.9 and 3.10 can be rewritten differently if the heat of adsorption varies from patch to patch (heterogeneous surface). The adsorption integration integral is given as: 
$\theta_{m}(p)=\int_{Q 1}^{Q 2} \theta_{l i}(p, Q) \delta(Q) d Q$

$\delta(Q)$ is the fraction of surface with heats between $Q$ and dQ. $\theta_{l i}(p, Q)$ is the local isotherm which varies with the partial pressure and the heat of adsorption. Equation 3.11 is written for a static and rigid homotattic patch.

However, when the surface is dynamic, general temporal variations in the fraction of surfaces, $\delta$, may be important. These temporal variations may be due to changes in surface area of each patch, while the total surface area is conserved or not conserved in the case of strong-metal-support-interactions (for metal catalysts). The dynamic surface where the fraction of surface with heats between $Q$ and $d Q$ is considered such that:

$\theta_{m}(p, t)=\int_{Q 1}^{Q 2} \theta_{l i}(p, Q) \delta(Q, t) d Q$

$\theta_{l i}(p, Q)$ is still represented by the Langmuir isotherm for a homotattic patch. Effects of lateral interactions (induced heterogeneity) can be used to modify $\theta_{l i}(p, Q) . \delta(Q, t)$ represents the temporal variation of the fraction of surfaces in each homotattic patch, which is also dependent on the heat of adsorption.

The above equation can be solved in two ways: either by obtaining the siteenergy distribution that corresponds to the overall adsorption integral equation (classII solutions) or using the observed adsorbed integral equation to obtain solutions of the site-energy-distribution (class-I). In class-I solutions, various isotherms have been observed: ${ }^{57}$ Freundlich Isotherm ${ }^{61}$, generalised Freundlich Isotherm ${ }^{62}$, LangmuirFreundlich Isotherm, Toth Isotherm ${ }^{63-65}$, Temkin-Pyzchev isotherm/Shlygin-Frumkin Isotherm ${ }^{66-69}$, Radke-Prausnitz-Jaroneic Isotherm ${ }^{70,71}$, Marczewski-Jaroniec Isotherm 72, Knowles-Moffat Isotherm ${ }^{73}, 74$, Dubinin-Radushkevich Isotherm ${ }^{75-78}$, DubininAstakhov Isotherm ${ }^{79}$, Freundlich-Dubinin-Radushkevich Isotherm ${ }^{80}$. In the class-I 
solutions, however, the variation with adlayer formation and restructuring that evolves with time cannot be considered.

This is represented in class-II solutions where the site-energy distribution is assumed, and the adsorption integral equation is produced for inclusion in statistical rate equations used in kinetic modelling. The site energy distributions are generally grouped into four categories: ${ }^{57}$ positive exponential, negative exponential (simple, truncated, modified, and hyperbolic), constant, Gaussian/skewed-Gaussian (normal, Log-normal, sinusoidal, Maxwell-Boltzmann, $y$-Type).

Considering, for instance, the skewed-Gaussian site-energy distribution, specifically the positive sinusoidal function that varies with time, equation 3.11 can be modified as:

$$
\delta(Q, t)=\sin ^{2}\left(\frac{\pi Q}{2 Q_{m}}\right) \int_{0}^{t} f(t) d t
$$

Where $Q_{m}$ is a general parameter characterising the distribution. $F(t)$ is a function that regulates the amplitude and phase behaviour of the sinusoidal wave representing the dynamic site-energy-distribution. The overall isotherm (representing the dynamic adsorption integral equation) is then given as:

$\theta_{m}(p, t)=\int_{0}^{t} f(t) d t \int_{Q 1}^{Q 2} \theta_{l i}(p, Q) \sin ^{2}\left(\frac{\pi Q}{2 Q_{m}}\right) d Q$

But $\theta_{m}$ represents one site for one specie.

Using the mass action formalism for a simple adsorption reaction, that is, $A+* \rightleftharpoons A *$, where * is denoted as a static active site, we obtain:

$\theta_{A, v}=K_{A} P_{A} \theta_{V}$

Where $\theta_{v}$ is the fraction of vacant sites available. For a homogeneous surface, onto which a local Langmuir isotherm is observed, we note that: 
$\theta_{m}(p)=\int_{Q 1}^{Q 2} \theta_{l i}(p, Q) \delta(Q) d Q$

As the heat of adsorption stays constant; $\theta_{m}(p)=\theta_{l i}(p)=\theta_{A}(p)$

Accounting for dynamics, the mass action formalism still remains as:

$\theta_{A}=K_{A} P_{A} \theta_{V}$ or $\theta_{A}=\frac{K_{A} P_{A}}{1+K_{A} P_{A}} ;$ where $\theta_{v}=1-\theta_{A}$

We further consider two scenarios for the temporal variation of the fraction of active sites on each patch in the homotattic patch model.

\subsection{Periodic surfaces}

With a sinusoidal dynamic belonging to the skewed/Gaussian or Gaussian distribution and a modulating time distribution, we obtain (Figure 5c):

$\theta_{m}(p, t)=\int_{0}^{t} f(t) d t \int_{Q 1}^{Q 2} \theta_{l i}(p, Q) \sin ^{2}\left(\frac{\pi Q}{2 Q_{m}}\right) d Q$

The time dependent $\int_{0}^{t} f(t) d t$ is due to varying patch surface areas, which allowing for a conserved total surface area should be used to define $\theta_{A, B}$ or $K_{A, B}$. The positive sinusoidal function is given in Figure $5 \mathrm{~d}$. Equation 3.18 highlights the physical description of the active site dynamics while the mass action law highlights chemical kinetic method. To merge both, we note that the chemical kinetic formalism would have to be modulated as:

$\theta_{A}=\frac{K_{A}(Q, t) P_{A}}{1+K_{A}(Q, t) P_{A}}$ or $\theta_{A}=K_{A}(Q, t) P_{A} \theta_{V}$ 


\subsection{Modified surfaces}

We represent $\theta_{A, B}$ as the coverage of $A$ on modified surface, B (Figure $5 e$ ). We note that for a multi-component specie in which the surface changes, the adsorption constant will be dependent on the surface (on the ith patch). This definition requires methods of measuring sublayer coverage which may be representative of adlayer or restructuring function. The physical description necessitates adsorption on changed surfaces. While the standard model uses:

$\theta_{A, v}=K_{A, v}(Q, t) P_{A} \theta_{V}$

where $\theta_{A, v}$ is the coverage of $\mathrm{A}$ on vacant sites. The coverage of $\mathrm{A}$ on surfaces that are modified by species B will be given as:

$\theta_{A, B}=K_{A, B}(Q, t) P_{A} \theta_{V}$

where $\theta_{V}$ is modified accordingly to $\theta_{v}=1-\theta_{A}-\theta_{B}-\theta_{A, B}$ (in the case of competitive sorption on both surfaces).

If two pseudo surfaces are involved, namely the "clean" surface and "modified" surface, with site conservation on both surfaces and in the case of non-competitive sorption on the "clean" surface we have:

$\theta_{v, s}=1-\theta_{A, v}-\theta_{B, v}$

On the "modified" surface, we have:

$$
\theta_{v, S S}=1-\theta_{A, B}-\theta_{B, B}
$$

We assume that there is no competition between surfaces such that each surface behaves independently. Thus, the adsorption constants are defined with respect to adsorption on a "clean" surface and "modified" surface (Figure 5e). This distinction allows for variation of the adsorption constants with time on stream, as the proportion of modified surface varies with reaction progress. The time integral 
$\int_{0}^{t} f(t) d t$ here is due to modified patches which evolves with reaction progress and used to define $\theta_{A, B}$ or $K_{A, B}$.

For a first order reaction of $A$ on a vacant site, the rate is given as:

$r=k_{a} K_{A, v}(Q, t) P_{A} \theta_{V}=k_{a} K_{A, v}(Q, t) P_{A} \theta_{V}$

For the reactions of $A$ on surfaces modified by $B$, the rate is given as:

$r=k_{a} K_{A, B}(Q, t) P_{A} \theta_{V}=k_{a} K_{A, B}(Q, t) P_{A} \theta_{V}$

The site-energy distribution is reflected in the variation of adsorption equilibrium constants with respect to process conditions and time on stream.

Mechanistic information on the catalytic action occurring on the catalyst surface can be obtained by kinetic studies or the direct study of the catalyst under operando working conditions. Langmuir ${ }^{81}$ postulated that the clean surface of a solid crystalline body must consist of atoms or molecules arranged in a surface lattice. The catalyst surface is regarded as containing elementary spaces where some spaces are vacant, and others are covered with adsorbed atoms or molecules. However, realistic depictions of catalyst surface are heterogeneous consisting of terraces, ledges, and kinks (TLK model). Taylor ${ }^{53}$ observed that only a small fraction of the surface is active which is sensitive to heat treatment and to the action of poisons, and the catalyst surface has a varying adsorption capacity. Further X-ray examination of metallic hydrogenation catalysts showed that there are groups of atoms in which the crystallization process is not complete. As opposed to flat clean surface observed by Langmuir, a degree of unsaturation is observed for atoms on the catalyst surface. In the Langmuir isotherm used for a homogeneous surface, the heat of adsorption stays constant with coverage. The heat of adsorption could exhibit various functional distributions with coverage including an exponential, ${ }^{82}$ or a linear distribution. ${ }^{83}$ 
Former consideration of the adsorption integral equations were based on invariant static patches. "Temporal/periodic" variation in patches has been considered by differing patch surface areas (Figure $5 \mathrm{c}$ ) while the total surface area is conserved or not conserved (in the case of strong metal support interactions for metals) and the evolution of "modified" surfaces (Figure 5d).

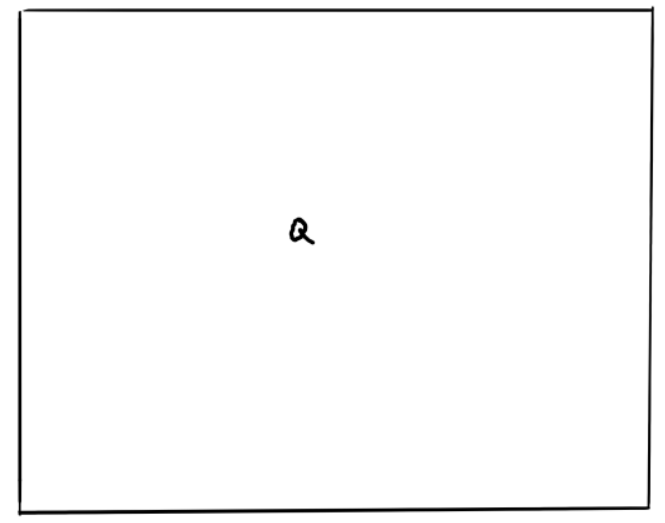

A. Homogeneous surface<smiles>[13CH3][13CH3]</smiles>

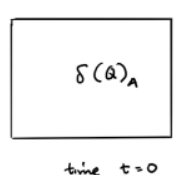

time $t=0$

Surfece B

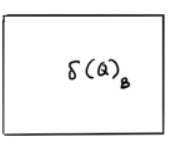

time $t=0$
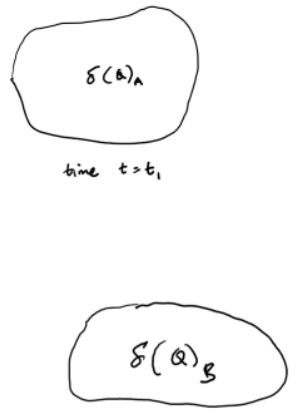

tive $t=t_{1}$

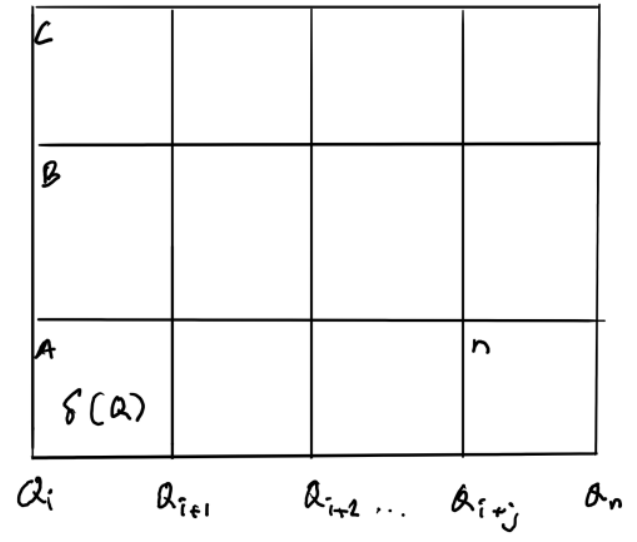

B. Heterogeneous surface with static sites
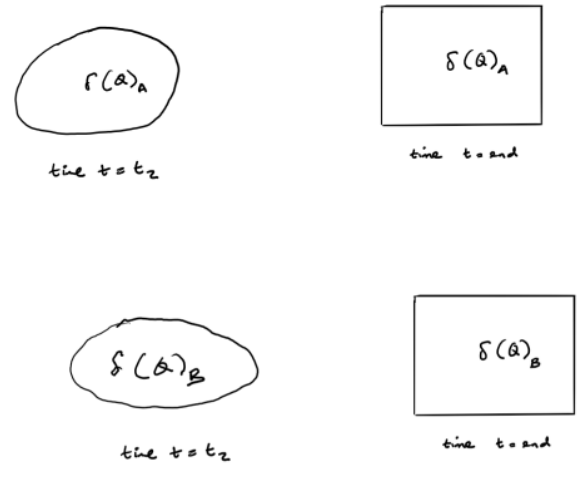

C. Heterogeneous surfaces with active site/surface dynamics (changing fraction of sites, while ensuring conservation of catalyst surface area)

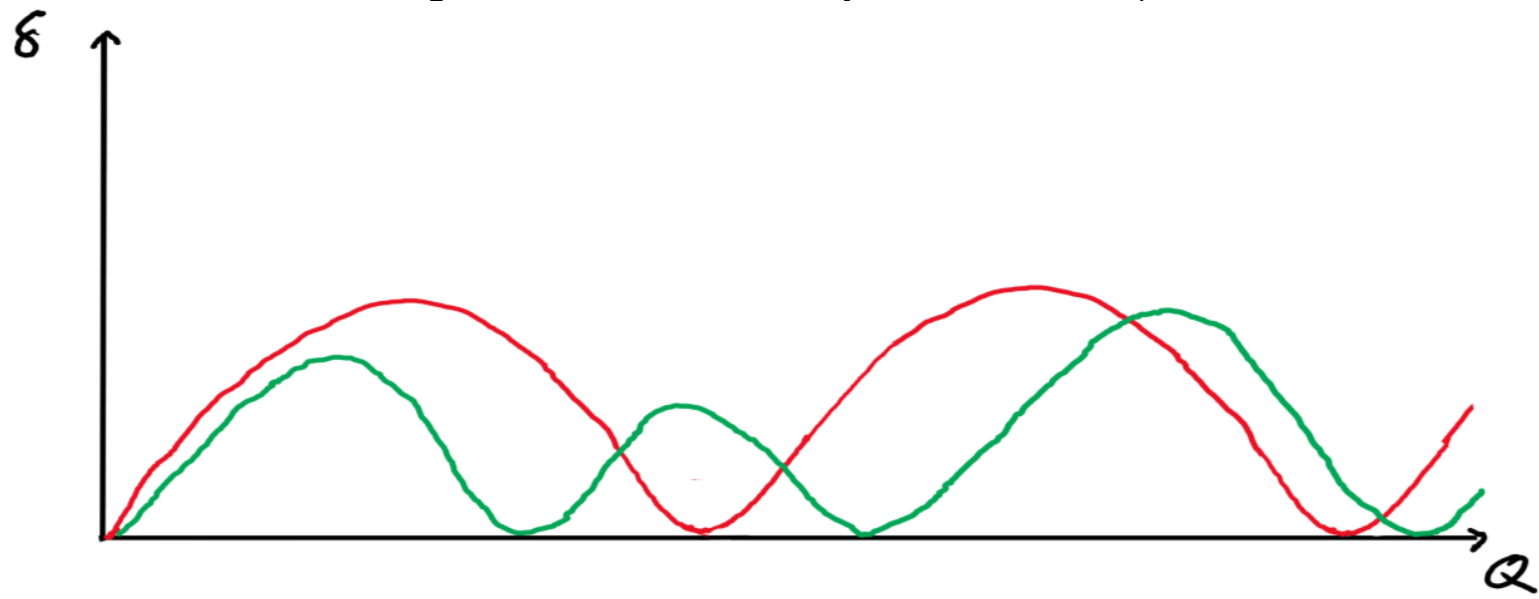


D. A positive sinusoidal function of the fraction of sites with heat of adsorption with constant amplitude and frequency representing a static surface (-). A dynamic surface is accounted for by changes in the fraction of sites with heat of adsorption at continuously changing amplitudes and frequencies (-)

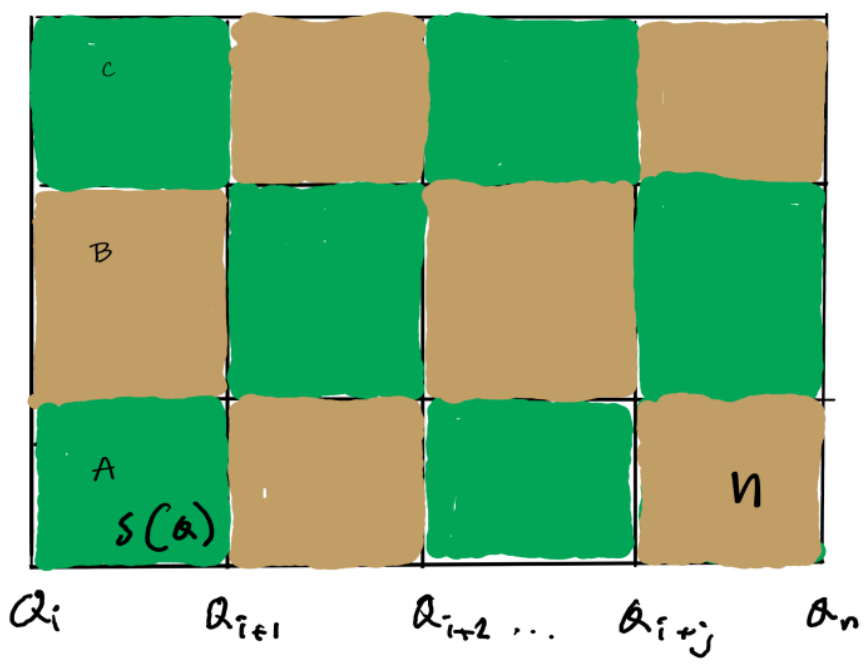

E. Heterogeneous surface with active site/surface dynamics (incorporating "clean (green)" surfaces and "modified (brown)" surfaces

Figure 5: Various constructs used in the formulation of the adsorption integral equation following the homotattic patch model. 


\section{Multiscale Microdynamic Models}

Boudart ${ }^{84}$ stated that: "the steady-state approximation can be considered as the most important general technique of applied chemical kinetics". A formal proof of this "hypothesis" that is applicable to all reaction mechanisms is not available because the rate equations for complex systems are often impossible to solve analytically. ${ }^{85}$ For a simple adsorption mechanism and with constant molar volume reactions where no expansion in the gas phase occurs, equations 4.1 and 4.2 are applicable:

Gas: $\varepsilon_{b} \frac{\partial C_{i, g}}{\partial t}=D_{i, g} \frac{\partial^{2} C_{i, g}}{\partial z^{2}}-u \frac{\partial C_{i, g}}{\partial z}-\Gamma_{t} S_{v}\left(1-\varepsilon_{b}\right)\left(k_{a} c_{i}-k_{d} \theta_{i Z}\right)$

Surface: $\frac{\partial \theta_{i, s}}{\partial t}=\frac{D_{i, s}}{r^{2}}\left(r^{2} \frac{\partial^{2} \theta_{i, g}}{\partial r^{2}}\right)+k_{a} c_{i}-k_{d} \theta_{i Z}$

where $\mathrm{k}_{\mathrm{a}}$ is the adsorption coefficient $\left(\mathrm{m}^{3} \mathrm{~mol}^{-1} \mathrm{~s}^{-1}\right), \mathrm{C}_{i}$ is the concentration of gas phase component, $\mathrm{i}\left(\mathrm{mol} \mathrm{m}^{-3}\right), \varepsilon_{b}$ is bed porosity $(-)$; $\mathrm{u}$ is the superficial velocity, $\left(\mathrm{m} \mathrm{s}^{-1}\right) ; \mathrm{z}$ is the bed length, $(\mathrm{m})$; $\mathrm{t}$ is time, $(\mathrm{s}) ; \Gamma_{t}$ is the concentration of active sites per unit surface area of catalyst $\left(\mathrm{mol} \mathrm{m}_{\mathrm{cat}^{-2}}{ }^{-2}\right) ; \mathrm{S}_{\mathrm{v}}$ is the catalyst surface area per unit volume $\left(\mathrm{m}_{\mathrm{cat}^{-1}}{ }^{-1}\right) ; k_{d}$ is the desorption rate coefficient $\left(\mathrm{s}^{-1}\right) ; \theta_{i}$ is the fractional surface coverage of the adsorbed specie; $D_{i, g}$ is the gas-phase dispersion coefficient $\left(m^{2} \mathrm{~s}^{-1}\right) ; D_{i, s}$ is the surface diffusivity $\left(\mathrm{m}^{2} \mathrm{~s}^{-1}\right) ; r$ is the radius of the catalyst pellet $(\mathrm{m})$.

For the scenario (section 3.2) where competitive surface adsorption on a modified surface occurs, equations 4.1 and 4.2 can be modified as:

$$
\begin{aligned}
& \text { Gas: } \varepsilon_{b} \frac{\partial C_{i, g}}{\partial t}=D_{i, g} \frac{\partial^{2} C_{i, g}}{\partial z^{2}}-u \frac{\partial C_{i, g}}{\partial z}-\Gamma_{t} S_{v}\left(1-\varepsilon_{b}\right)\left(k_{a} c_{i}-k_{d} \theta_{i Z}-k_{d} \theta_{i, j}\right) \\
& \text { Surface: } \frac{\partial \theta_{i, s}}{\partial t}=\frac{D_{i, s}}{r^{2}}\left(r^{2} \frac{\partial^{2} \theta_{i, g}}{\partial r^{2}}\right)+k_{a} c_{i}-k_{d} \theta_{i Z}-k_{d} \theta_{i, j}
\end{aligned}
$$


The following equations can be further modified if the concentration of active sites per unit surface area also changes in accordance with active site dynamics (Section 3.1). A linear change is expressed as:

$\Gamma_{t}=\Gamma_{t, o}-\alpha t$

Where $\alpha$ is a parameter characterising the linear variation and " $\mathrm{t}$ " is time on stream. A logarithmic variation is given as:

$$
\Gamma_{t}=\Gamma_{t, o}-\beta \ln t
$$

Where $\beta$ is a parameter characterising the logarithmic variation.

This set of equations can be reduced (by using the steady-state approximation) to the conventional differential-algebraic-equations provided by Dumesic and coworkers. ${ }^{86}$ Equations 4.3 and 4.4 could however be solved without these assumptions where the variation of gas species with reactor bed length and time on stream is provided.

A realistic kinetic model for selective oxidation reactions should include the inclusion of adsorption constants that vary over fresh, activated and working catalysts at specified times on stream ("clean", "modified" and "periodic" surfaces) and the specification of the distribution of metal-oxygen species as initial conditions for catalyst state as a result of the oxygen adsorption deconvolution and its modulation.

Catalyst states could be obtained as a function of the local chemical potentials of oxygen and steam leading to restructuring. The catalyst state is represented by the number density of surface vacancies. If $[*]_{O}$ represents the number density of all adsorption sites (vacant and occupied) on a catalyst surface, then:87

$$
\begin{aligned}
& {[*]_{O}=[*]+[i *]} \\
& \theta_{i}=\frac{[i *]}{[*]_{O}}
\end{aligned}
$$


$[*]$ represents vacant sites, $[i *]$ represents sites occupied by "i". $\theta_{i}$ is the fractional surface coverage defined as the fraction of total surface adsorption sites that are occupied by "i”.

The number density of surface vacancies [*] i.e., catalyst state, varies along the bed length due to different chemical potentials and "gas-mediated" dispersive factors. Consequently, the following equations are obtained:

Gas: $\varepsilon_{b} \frac{\partial C_{i, g}}{\partial t}=D_{i, g} \frac{\partial^{2} C_{i, g}}{\partial z^{2}}-u \frac{\partial C_{i, g}}{\partial z}-\Gamma_{t} S_{v}\left(1-\varepsilon_{b}\right)\left(k_{a} c_{i}-k_{d} \theta_{i z}-k_{d} \theta_{i, j}\right)$

Surface: $\frac{\partial \theta_{i, s}}{\partial t}=\frac{D_{i, s}}{r^{2}}\left(r^{2} \frac{\partial^{2} \theta_{i, s}}{\partial r^{2}}\right)+k_{a} c_{i}-k_{d} \theta_{i z}-k_{d} \theta_{i, j}$

Site balance: $[*]_{O}=[*]+[i *]$

Catalyst State: $\frac{d \theta_{L}}{d t}=\frac{D_{i, L}}{r^{2}}\left(r^{2} \frac{\partial^{2} \theta_{i, L}}{\partial r^{2}}\right)+f\left(P_{i}, \theta_{i}, T_{i}\right)$

Selective oxidation catalysis involves an oxygen reduction mechanism with electron transfer to the catalyst surface. The physical principles governing oxidation catalysis sits at the corridor between combustion physics and semiconductor physics. To account for electron transfer, a set of physics-based models first presented by Van Overstraeten are tenable: ${ }^{88,89}$

Poisson equation: The Poisson equation is essentially the third Maxwell equation and is valid for all materials with a time dependent permittivity. $\varepsilon$ is permittivity, $q$ is the elementary charge, $p$ is the positively charged hole concentration, $n$ is the negatively charged electron concentration and $C$ is an additional concentration, $\varphi$ is the electrostatic potential.

$\operatorname{div} \operatorname{grad} \varphi=\frac{q}{\varepsilon} \cdot(n-p-C)$ 


\section{Continuity equations for electrons}

The conduction current density $\vec{J}$ can be split into a component caused by electrons, $\overrightarrow{J_{n}}$ and a component caused by holes, $\overrightarrow{J_{p}}$. In 4.11 and 4.12 , it is further assumed that all charges, except the mobile carrier electrons and holes are time invariant. The influence of charged defects, e.g., vacancies, dislocation, deep recombination traps, which may change their charge state with time are negligible $\left(\frac{\partial C}{\partial t}=0\right)$ $\operatorname{div} \overrightarrow{J_{n}}-q \cdot \frac{\partial n}{\partial t}=q \cdot R$

\section{Continuity equations for holes}

$\operatorname{div} \overrightarrow{J_{p}}+q \cdot \frac{\partial p}{\partial t}=q \cdot R$

"R" can be understood as a function describing the net generation or recombination of electrons or holes. Positive $\mathrm{R}$ means recombination and negative $\mathrm{R}$ means generation.

\section{Current relations for electrons}

$\overrightarrow{J_{n}}=q \cdot n \cdot \mu_{n} \cdot \overrightarrow{E_{n}}+q \cdot \mathrm{D}_{n} \cdot \operatorname{grad} n$

\section{Current relations for holes}

$\overrightarrow{J_{p}}=q \cdot p \cdot \mu_{p} \cdot \overrightarrow{E_{p}}-q \cdot \mathrm{D}_{p} \cdot \operatorname{grad} p$

Where $\mu_{n}$ and $\mu_{p}$ are effective carrier mobilities for electrons and holes respectively. $\overrightarrow{E_{n}}$ and $\overrightarrow{E_{p}}$ are the electric field for the drift current components of electron and hole current density, respectively. $D_{n}$ and $D_{p}$ are the diffusion constants for electrons and holes, respectively. 
By substituting the current relations for electrons and holes into the Poisson equation and the continuity equations for electrons and holes, we obtain a system of three partial differential equations with the dependent variables $\varphi, n, p$.

\section{Poisson equation}

$\operatorname{div} \operatorname{grad} \varphi-\frac{q}{\varepsilon} \cdot(n-p-C)=0$

\section{Continuity equation for electrons}

$\operatorname{div}\left(D_{n} \cdot \operatorname{grad} n-\mu_{n} \cdot n \cdot \operatorname{grad} \varphi\right)-R(\varphi, n, p)=\frac{\partial n}{\partial t}$

\section{Continuity equation for holes}

$\operatorname{div}\left(D_{p} \cdot \operatorname{grad} p+\mu_{p} \cdot p \cdot \operatorname{grad} \varphi\right)-R(\varphi, n, p)=\frac{\partial p}{\partial t}$

To solve the charge equations using differential equations, "div" and "grad" in the basic equations are written explicitly:

\section{Poisson equation}

$\lambda^{2} \cdot\left(\frac{\partial^{2} \varphi}{\partial x^{2}}+\frac{\partial^{2} \varphi}{\partial y^{2}}\right)-n+p+C=0$

\section{Continuity equations for electrons}

$\frac{\partial}{\partial x}\left(D_{n} \cdot \frac{\partial n}{\partial x}-\mu_{n} \cdot n \cdot \frac{\partial \varphi}{\partial x}\right)+\frac{\partial}{\partial y}\left(D_{n} \cdot \frac{\partial n}{\partial y}-\mu_{n} \cdot n \cdot \frac{\partial \varphi}{\partial y}\right)-R(\varphi, n, p)=0$

\section{Continuity equations for holes}

$\frac{\partial}{\partial x}\left(D_{p} \cdot \frac{\partial p}{\partial x}-\mu_{p} \cdot p \cdot \frac{\partial \varphi}{\partial x}\right)+\frac{\partial}{\partial y}\left(D_{p} \cdot \frac{\partial p}{\partial y}-\mu_{p} \cdot n \cdot \frac{\partial \varphi}{\partial y}\right)-R(\varphi, n, p)=0$

Heat flow equation (for non-isothermal reactor conditions)

$\rho \cdot c \cdot \frac{\partial T}{\partial t}-H=\operatorname{div} k(T) \cdot \operatorname{grad} T$

Specific to equation 4.15 is the following nomenclature: $\rho$ and $c$ are the specific mass density and specific heat of the material. $\mathrm{T}$ is temperature, $\mathrm{t}$ is time. $\mathrm{K}(\mathrm{T})$ and $\mathrm{H}$ denote the thermal conductivity and the locally generated heat. 
Equations 4.3 to 4.15 represent a multiscale model for heterogeneous catalysis which considers the evolution of gas species, adsorbed species, catalyst states, and charge transport with catalyst bed length and time on stream. 


\section{Conclusions}

In order to account for microscopic and macroscopic factors governing selectivity, a multiscale model is presented to extract descriptors and correlations. Active site dynamics is considered by introducing open catalytic cycles. Catalyst surface dynamics is accounted for by incorporating the homotattic patch model. Semiconductor physics is used in incorporating the effects of charge transport. Adspecie accumulation due to surface diffusion and reactivity is considered. Catalyst state variation due to the changing local chemical potential along the reactor bed length and the temporal and spatial evolution of gaseous species are considered. The multiscale models are useful for simulating transient, steadystate, and deactivation kinetics during heterogeneous catalysis to obtain catalyst activity and lifetime, as well as product selectivity descriptors. 


\section{Declarations of Competing Interest}

There are no competing financial interests or personal relationships that could have appeared to influence the work reported in this paper.

\section{References}

1. Su, D. S.; Zhang, B.; Schlögl, R., Electron Microscopy of Solid CatalystsTransforming from a Challenge to a Toolbox. Chem. Rev. 2015, 115 (8), 28182882.

2. Frenken, J. W. M.; Groot, I. M. N., Seeing dynamic phenomena with live scanning tunneling microscopy. MRS Bull. 2017, 42 (11), 834-841.

3. Meirer, F.; Weckhuysen, B. M., Spatial and temporal exploration of heterogeneous catalysts with synchrotron radiation. Nature Reviews Materials 2018, 3 (9), 324-340.

4. Vamvakeros, A.; Jacques, S. D. M.; Di Michiel, M.; Matras, D.; Middelkoop, V.; Ismagilov, I. Z.; Matus, E. V.; Kuznetsov, V. V.; Drnec, J.; Senecal, P.; Beale, A. M., 5D operando tomographic diffraction imaging of a catalyst bed. Nature Communications 2018, 9 (1), 4751.

5. De Vrieze, J. E.; Gunasooriya, G. T. K. K.; Thybaut, J. W.; Saeys, M., Operando computational catalysis: shape, structure, and coverage under reaction conditions. Current Opinion in Chemical Engineering 2019, 23, 85-91.

6. Oyama, S. T.; Somorjai, G. A., Effect of structure in selective oxide catalysis: Oxidation reactions of ethanol and ethane on vanadium oxide. J. Phys. Chem. 1990, 94 (12), 5022-5028.

7. Spencer, N. D.; Schoonmaker, R. C.; Somorjai, G. A., J. Catal. 1982, 74 (129).

8. Dumesic, J. A.; Topsøe, H.; Boudart, M., Surface, catalytic and magnetic properties of small iron particles. III. Nitrogen induced surface reconstruction. J. Catal. 1975, 37 (3), 513-522.

9. Joo, S. H.; Park, J. Y.; Renzas, J. R.; Butcher, D. R.; Huang, W.; Somorjai, G. A., Size effect of ruthenium nanoparticles in catalytic carbon monoxide oxidation. Nano Lett. 2010, 10 (7), 2709-2713.

10. Ryder, J. A.; Chakraborty, A. K.; Bell, A. T., Density functional theory study of proton mobility in zeolites: Proton migration and hydrogen exchange in ZSM-5. J. Phys. Chem. B 2000, 104 (30), 6998-7011.

11. Somorjai, G. A.; Park, J. Y., Molecular factors of catalytic selectivity. Angewandte Chemie - International Edition 2008, 47 (48), 9212-9228.

12. Thomas, J. M.; Thomas, W. J., Principles and Practice of Heterogeneous Catalysis 2nd ed.; Wiley-VCH: Weinheim, Germany, 2015; p 744.

13. van Santen, R.; Neurock, M., Molecular Heterogeneous Catalysis: A Conceptual and Computational Approach. Wiley-VCH Verlag GmbH: 2006.

14. Grasselli, R. K., Fundamental principles of selective heterogeneous oxidation catalysis. Top. Catal. 2002, 21 (1-3), 79-88.

15. Schlögl, R., Heterogeneous Catalysis. Angew. Chem. Int. Ed. 2015, 54 (11), 3465-3520.

16. Decarolis, D.; Clark, A. H.; Pellegrinelli, T.; Nachtegaal, M.; Lynch, E. W.; Catlow, C. R. A.; Gibson, E. K.; Goguet, A.; Wells, P. P., Spatial Profiling of a 
$\mathrm{Pd} / \mathrm{Al} 2 \mathrm{O} 3$ Catalyst during Selective Ammonia Oxidation. ACS Catalysis 2021, 2141-2149.

17. Serrer, M.-A.; Stehle, M.; Schulte, M. L.; Besser, H.; Pfleging, W.; Saraçi, E.; Grunwaldt, J.-D., Spatially-Resolved Insights Into Local Activity and Structure of Ni-based $\mathrm{CO} 2$ Methanation Catalysts in Fixed-bed Reactors. ChemCatChem 2021, n/a (n/a).

18. Wolke, F.; Hu, Y.; Schmidt, M.; Korup, O.; Horn, R.; Reichelt, E.; Jahn, M.; Michaelis, A., Spatially-resolved reaction profiles in Fischer-Tropsch synthesis - influence of operating conditions and promotion for iron-based catalysts. Catal. Commun. 2021, 158, 106335.

19. Liu, P.; Nørskov, J. K., Ligand and ensemble effects in adsorption on alloy surfaces. Physical Chemistry Chemical Physics 2001, 3 (17), 3814-3818.

20. Nørskov, J. K.; Bligaard, T.; Hvolbæk, B.; Abild-Pedersen, F.; Chorkendorff, I.; Christensen, C. H., The nature of the active site in heterogeneous metal catalysis. Chem. Soc. Rev. 2008, 37 (10), 2163-2171.

21. Kim, M.; Bertram, M.; Pollmann, M.; von Oertzen, A.; Mikhailov, A. S.; Rotermund, H. H.; Ertl, G., Controlling chemical turbulence by global delayed feedback: pattern formation in catalytic $\mathrm{CO}$ oxidation on $\mathrm{Pt}(110)$. Science 2001, 292 (5520), 1357-60.

22. Arán-Ais, R. M.; Rizo, R.; Grosse, P.; Algara-Siller, G.; Dembélé, K.; Plodinec, M.; Lunkenbein, T.; Chee, S. W.; Cuenya, B. R., Imaging electrochemically synthesized $\mathrm{Cu} 2 \mathrm{O}$ cubes and their morphological evolution under conditions relevant to $\mathrm{CO} 2$ electroreduction. Nature Communications 2020, 11 (1), 3489.

23. Plodinec, M.; Nerl, H. C.; Farra, R.; Willinger, M. G.; Stotz, E.; Schlögl, R.; Lunkenbein, T., Versatile Homebuilt Gas Feed and Analysis System for Operando TEM of Catalysts at Work. Microsc. Microanal. 2020, 1-9.

24. Barroo, C.; Wang, Z. J.; Schlögl, R.; Willinger, M. G., Imaging the dynamics of catalysed surface reactions by in situ scanning electron microscopy. Nature Catalysis 2020, 3 (1), 30-39.

25. Masliuk, L.; Heggen, M.; Noack, J.; Girgsdies, F.; Trunschke, A.; Hermann, K. E.; Willinger, M. G.; Schlögl, R.; Lunkenbein, T., Structural Complexity in Heterogeneous Catalysis: Cataloging Local Nanostructures. Journal of Physical Chemistry C 2017, 121 (43), 24093-24103.

26. Sanfiz, A. C.; Hansen, T. W.; Teschner, D.; Schnörch, P.; Girgsdies, F.; Trunschke, A.; Schlögl, R.; Looi, M. H.; Hamid, S. B. A., Dynamics of the MoVTeNb oxide M1 phase in propane oxidation. Journal of Physical Chemistry C 2010, 114 (4), 1912-1921.

27. Trunschke, A.; Noack, J.; Trojanov, S.; Girgsdies, F.; Lunkenbein, T.; Pfeifer, V.; Havecker, M.; Kube, P.; Sprung, C.; Rosowski, F.; Schlogl, R., The Impact of the Bulk Structure on Surface Dynamics of Complex Mo-V-based Oxide Catalysts. Acs Catalysis 2017, 7 (4), 3061-3071.

28. Li, X.; Lunkenbein, T.; Pfeifer, V.; Jastak, M.; Nielsen, P. K.; Girgsdies, F.; Knop-Gericke, A.; Rosowski, F.; Schlögl, R.; Trunschke, A., Selective Alkane Oxidation by Manganese Oxide: Site Isolation of MnOx Chains at the Surface of MnWO4 Nanorods. Angewandte Chemie - International Edition 2016, 55 (12), 4092-4096.

29. Sprenger, P.; Kleist, W.; Grunwaldt, J. D., Recent Advances in Selective Propylene Oxidation over Bismuth Molybdate Based Catalysts: Synthetic, 
Spectroscopic, and Theoretical Approaches. ACS Catalysis 2017, 7 (9), 56285642.

30. Imbihl, R.; Ertl, G., Oscillatory Kinetics in Heterogeneous Catalysis. Chem. Rev. 1995, 95 (3), 697-733.

31. Kobayashi, H.; Masayoshi, k., Transient response method in heterogeneous catalysis. Catalysis Reviews 1974, 10 (1), 139-176.

32. Kobayashi, M., Characterization of transient response curves in heterogeneous catalysis-I Classification of the curves. Chem. Eng. Sci. 1982, 37 (3), 393-401.

33. Kobayashi, M., Characterization of transient response curves in heterogeneous catalysis - 2. Estimation of the reaction mechanism in the oxidation of ethylene over a silver catalyst from the mode of the transient response curves. Chem. Eng. Sci. 1982, 37 (3), 403-409.

34. Kobayashi, M.; Maeda, Y.; Takahashi, N., Discrimination of rival kinetic models in heterogeneous catalysis by the dynamic behaviour of products. Journal of Chemical Technology and Biotechnology, Chemical Technology 1983, 33 A (4), 219-226.

35. Ardagh, M. A.; Abdelrahman, O. A.; Dauenhauer, P. J., Principles of Dynamic Heterogeneous Catalysis: Surface Resonance and Turnover Frequency Response. ACS Catalysis 2019, 9 (8), 6929-6937.

36. Gopeesingh, J.; Ardagh, M. A.; Shetty, M.; Burke, S. T.; Dauenhauer, P. J.; Abdelrahman, O. A., Resonance-Promoted Formic Acid Oxidation via Dynamic Electrocatalytic Modulation. ACS Catalysis 2020, 10 (17), 9932-9942.

37. Bennett, C. O., Understanding Heterogeneous Catalysis Through the Transient Method. In Catalysis Under Transient Conditions, American Chemical Society: 1982; Vol. 178, pp 1-32.

38. Bennett, C. O., Experiments and Processes in the Transient Regime for Heterogeneous Catalysis. In Advances in Catalysis, Haag, W. O.; Gates, B. C.; Knözinger, H., Eds. Academic Press: 1999; Vol. 44, pp 329-416.

39. Boudart, M., Kinetics on ideal and real surfaces. AlChE J. 1956, 2 (1), 62-64.

40. Djéga-Mariadassou, G.; Boudart, M., Classical kinetics of catalytic reactions. J. Catal. 2003, 216 (1-2), 89-97.

41. Warshel, A., Dynamics of enzymatic reactions. Proceedings of the National Academy of Sciences 1984, 81 (2), 444-448.

42. Warshel, A.; Bora, R. P., Perspective: Defining and quantifying the role of dynamics in enzyme catalysis. J. Chem. Phys. 2016, 144 (18).

43. Warshel, A., Multiscale modeling of biological functions: From enzymes to molecular machines (nobel lecture). Angewandte Chemie - International Edition 2014, 53 (38), 10020-10031.

44. Pisliakov, A. V.; Cao, J.; Kamerlin, S. C.; Warshel, A., Enzyme millisecond conformational dynamics do not catalyze the chemical step. Proc Natl Acad Sci U S A 2009, 106 (41), 17359-64.

45. Kamerlin, S. C. L.; Warshel, A., At the dawn of the 21st century: Is dynamics the missing link for understanding enzyme catalysis? Proteins 2010, 78 (6), 1339-1375.

46. Masliuk, L.; Schmidt, F.-P.; Hetaba, W.; Plodinec, M.; Auffermann, G.; Hermann, K.; Teschner, D.; Girgsdies, F.; Trunschke, A.; Schlögl, R.; Lunkenbein, T., Compositional Decoupling of Bulk and Surface in OpenStructured Complex Mixed Oxides. The Journal of Physical Chemistry C 2020, 124 (42), 23069-23077. 
47. Trunschke, A.; Bellini, G.; Boniface, M.; Carey, S. J.; Dong, J. H.; Erdem, E.; Foppa, L.; Frandsen, W.; Geske, M.; Ghiringhelli, L. M.; Girgsdies, F.; Hanna, R.; Hashagen, M.; Havecker, M.; Huff, G.; Knop-Gericke, A.; Koch, G.; Kraus, P.; Krohnert, J.; Kube, P.; Lohr, S.; Lunkenbein, T.; Masliuk, L.; d'Alnoncourt, R. N.; Omojola, T.; Pratsch, C.; Richter, S.; Rohner, C.; Rosowski, F.; Ruther, F.; Scheffler, M.; Schlogl, R.; Tarasov, A.; Teschner, D.; Timpe, O.; Trunschke, P.; Wang, Y. Q.; Wrabetz, S., Towards Experimental Handbooks in Catalysis. Top. Catal. 2020, 63 (19-20), 16831699.

48. Wulff, G., On the question of speed of growth and dissolution of crystal surfaces. Z Krystallogr. Mineral 1901, 34 (5/6), 449-530.

49. Wulff, G., XXV. Zur Frage der Geschwindigkeit des Wachsthums und der Auflösung der Krystallflächen. Zeitschrift für Kristallographie - Crystalline Materials 1901, 34 (1-6), 449-530.

50. van Santen, R.; Niemantsverdriet, J. W., Chemical Kinetics and Catalysis. 1995.

51. Boudart, M.; Djega-Mariadassou, G., Kinetics of heterogeneous catalytic reactions Princeton University Press: Princeton, 1984.

52. Newton, I., De analysi per aequationes numero terminorum infinitas. 1744.

53. Taylor, H. S., A theory of the catalytic surface. Proceedings of the Royal Society of London. Series A, Containing Papers of a Mathematical and Physical Character 1925, 108 (745), 105-111.

54. Stranski, I. N., Z. Phys. Chem 1928, 11.

55. Kossel, W., Nach. Ges. Wiss. 1957, 135.

56. Hirth, J. P.; Pound, G. M., Condensation and Evaporation, Nucleation and Growth Kinetics. Pergamon Press, Oxford: 1963.

57. Doraiswamy, L. K., Catalytic reactions and reactors: A surface science approach. Prog. Surf. Sci. 1991, 37 (1-4), 1-277.

58. Fowler, R. H.; Guggenheim, E. A., Statistical Thermodynamics. Cambridge University Press: Cambridge, 1949.

59. Everett, D. H., The thermodynamics of adsorption. Part II.-Thermodynamics of monolayers on solids. Transactions of the Faraday Society 1950, 46 (0), $942-$ 957.

60. Sanford, C.; Ross, S., Homotattic Surface-A Suggested New Word. The Journal of Physical Chemistry 1954, 58 (3), 288-288.

61. Zeldowitsch, J., Adsorption site energy distribution. Acta Physicochim. URSS 1934, 1, 961-973.

62. Sips, R., On the structure of a catalyst surface. II. The Journal of Chemical Physics 1950, 18 (8), 1024-1026.

63. Tóth, J., Acta Chimica (Academiae Scientiarum) Hungaricae. 1962, 32, 31-32.

64. Toth, J., Acta Chim. Hung. 1971, 69.

65. Toth, J., Proc. Int. Conf. Colloid and Surface Sci. 1975, 41.

66. Temkin, M.; Pyzhev, V., Acta Physicochim. URSS 1940, 12.

67. Temkin, M.; Levich, V., Zh. Fiz. Khim. 1946, 12.

68. Shlygin, A.; Frumkin, A., Acta Physicochim. URSS 1935, 3.

69. Temkin, M. I., Adsorption equilibrium and the kinetics of processes on nonhomogeneous surfaces and in the interaction between adsorbed molecules. Zh. Fiz. Khim. 1941, 15 (3), 296-332.

70. Jaroniec, M., Physical adsorption on heterogeneous solids. Adv. Colloid Interface Sci. 1983, 18 (3-4), 149-225. 
71. Radke, C. J.; Prausnitz, J. M.; Radke, C. J., Adsorption of Organic Solutes from Dilute Aqueous Solution on Activated Carbon. Ind. Eng. Chem. Fundam. 1972, 11 (4), 445-451.

72. Marczewski, A. W.; Jaroniec, M., A new isotherm equation for single-solute adsorption from dilute solutions on energetically heterogeneous solids - Short communication. Monatshefte für Chemie - Chemical Monthly 1983, 114 (6-7), 711-715.

73. Jaroniec, M., Adsorption on heterogeneous surfaces: The exponential equation for the overall adsorption isotherm. Surf. Sci. 1975, 50 (2), 553-564.

74. Knowles, A. J.; Moffat, J. B., The physical adsorption of gases on boron phosphate. II. Argon. J. Colloid Interface Sci. 1972, 41 (1), 116-123.

75. Hobson, J. P.; Armstrong, R. A., A study of physical adsorption at very low pressures using ultrahigh vacuum techniques. J. Phys. Chem. 1963, 67 (10), 2000-2008.

76. Sokolowski, S., Vuoto 1975, 8.

77. Misra, D. N., Adsorption on heterogeneous surfaces: A dubinin-radushkevich equation. Surf. Sci. 1969, 18 (2), 367-372.

78. Dubinin, M. M.; Radushkevich, L. V., The equation of the characteristic curve of the activated charcoal. Proc. Acad. Sci. USSR Phys. Chem. Sect. 1947, 55, 331-337.

79. Dubinin, M. M., Prog. in Surface and Membrane Sci 1975, 9, 1-70.

80. Cerofolini, G. F., The theory of physisorption on real solid surfaces. J. Low Temp. Phys. 1976, 23 (5-6), 687-697.

81. Langmuir, I., Part II - "Heterogeneous reactions": Chemical reactions on surfaces. Transactions of the Faraday Society 1922, 17, 607-620.

82. Freundlich, H., Colloids and Capillary Chemistry. Metheun: London, 1926.

83. Frumkin, A.; Slygin, A., Acta Physiochimica U.R.S.S 1935, 3, 791.

84. Boudart, M., Kinetics of Catalytic Processes. Prentice-Hall: Englewood Cliffs, NJ, 1968.

85. Vannice, M. A., Kinetics of Catalytic Reactions. Springer: 2005.

86. Motagamwala, A. H.; Ball, M. R.; Dumesic, J. A., Microkinetic Analysis and Scaling Relations for Catalyst Design. Annu Rev Chem Biomol Eng 2018, 9 (1), 413-450.

87. Davis, M. E.; Davis, R. J., Fundamentals of Chemical Reaction Engineering McGraw-Hill: New York, 2003; p 368.

88. Overstraeten, R. J. V.; DeMan, H. J.; Mertens, R. P., Transport equations in heavy doped silicon. IEEE Transactions on Electron Devices 1973, 20 (3), 290298.

89. Selberherr, S., Analysis and Simulation of Semiconductor Devices. 1984. 